\title{
Comparative Study between Intraoperative Frozen Section and Scrape Smear Cytology in the Diagnosis of Ovarian Neoplasm
}

\author{
Amany M. Abdelghany, Essa M. Arafa, Nadia M. Madkour, Wael S. Nossair*, \\ Ekramy A. Mohamed, Walid A. Abdelsalam, Amira A. Salem \\ Obstetrics \& Gynecology Department, Faculty of Medicine, Zagazig University, Zagazig, Egypt \\ Email: ${ }^{*}$ wsnossair@gmail.com
}

Received 20 October 2014; revised 22 November 2014; accepted 1 December 2014

Academic Editor: Yasam Kemal Akpak, Ankara Mevki Military Hospital, Turkey

Copyright (C) 2015 by authors and Scientific Research Publishing Inc.

This work is licensed under the Creative Commons Attribution International License (CC BY). http://creativecommons.org/licenses/by/4.0/

(c) $\underset{\text { EY }}{0}$ Open Access

\section{Abstract}

Objective: The aim of this work is to compare between intraoperative frozen section and scrape smear cytology in the diagnosis of ovarian neoplasm. Method: This study was performed between March 2011 and March 2012, on 50 patients presented with ovarian mass. Gross examination of the tumor removed was done by inspection and palpation. The specimen was then cut with a sharp knife into two halves. The area was scraped with a sharp scalpel or the end of a glass slide, depending upon the type of tissue. A semifluid drop thus obtained was spread over a glass slide. One to four slides per case were taken from different representative areas. The slides were labelled and immediately put into $95 \%$ ethyl alcohol and stained with hematoxylin-eosin. The specimens were then fixed in formalin. Paraffin blocks of the sections were processed in the routine way and sections were stained with hematoxylin and eosin ( $\mathrm{H}$ and $\mathrm{E}$ ). Assessment of the overall accuracy of the intraoperative diagnosis was classified as concordant or discordant. Results: The sensitivity, specificity, positive predictive value, negative predictive value and accuracy of scraping technique in the diagnosis of benign ovarian masses were $100 \%, 95.2 \%, 96.7 \%, 100 \%$ and $98 \%$ respectively. The sensitivity, specificity, positive predictive value, negative predictive value and accuracy of scraping technique in the diagnosis of border line ovarian masses were $100 \%, 93.4 \%, 25 \%, 100 \%$ and $94 \%$ respectively. The sensitivity, specificity, positive predictive value, negative predictive value and accuracy of scraping technique in the diagnosis of malignant ovarian masses were $80 \%$, $100 \%, 100 \%, 88.2 \%$ and $92 \%$ respectively. The sensitivity, specificity, positive predictive value, negative predictive value and accuracy of frozen section in the diagnosis of benign ovarian masses were $100 \%, 100 \%, 100 \%, 100 \%$ and $100 \%$ respectively. The sensitivity, specificity, positive predictive value, negative predictive value and accuracy of frozen section in the diagnosis of borderline

\footnotetext{
Corresponding author.
}

How to cite this paper: Abdelghany, A.M., et al. (2015) Comparative Study between Intraoperative Frozen Section and Scrape Smear Cytology in the Diagnosis of Ovarian Neoplasm. Open Journal of Obstetrics and Gynecology, 5, 28-35. 
ovarian masses were $100 \%, 95.9 \%, 33.3 \%, 100 \%$ and $96 \%$ respectively. The sensitivity, specificity, positive predictive value, negative predictive value and accuracy of frozen section in the diagnosis of malignant ovarian masses were $90 \%, 100 \%, 100 \%, 93.8 \%$ and $96 \%$ respectively. Conclusion: Frozen section is more accurate than smear preparations in the intraoperative assessment of ovarian tumors in this study. However, the cytology preparations were helpful in supporting the histological diagnoses, and in some cases, provided additional useful information. Thus, cytology has a complementary role to frozen section in the intraoperative assessment of ovarian lesions. At the centers where the facilities of frozen section are not available, intraoperative scrape cytology is a useful tool for intraoperative diagnosis of tumor.

\section{Keywords}

\section{Frozen Section, Intraoperative Assessment, Scrape Smear Cytology, Ovarian}

\section{Introduction}

Ovarian neoplasms are a heterogeneous group of benign and malignant tumors of epithelial, stromal and germcell origin. Histopathology of a paraffin section remains the ultimate gold standard in tissue diagnosis [1].

Rapid diagnosis of surgically removed specimens has created many controversies and a single completely reliable method has not yet been developed. Frozen section is routinely used by the surgical pathology laboratories for intra-operative diagnosis [2]. Intra-operative diagnosis of ovarian lesions can be achieved by a number of cytological techniques, including imprint and scrape cytology, and intra-operative fine-needle aspiration cytology (FNAC). These methods are less accurate as compared to frozen section [3]. However, few studies have demonstrated that in experienced hands the diagnostic efficacy of intra-operative cytology is comparable to that of frozen section with $92 \%$ diagnostic accuracy in characterizing cytological pattern and morphology of ovarian tumors [4].

Intra-operative cytology has high accuracy rates, excellent preservation of cellular details, and the possibility of identifying focal, macroscopically undetectable neoplastic lesion in large tissue fragments. The method is simple and inexpensive, not requiring special technique or instrument. The disadvantages of intraoperative cytology are very few and high accuracy rates can be achieved with experience. It is though not possible to distinguish in situ from infiltrating carcinoma and to evaluate the depth of invasion and/or margins of resection. Thus, apart from its diagnostic role, intraoperative cytology can become a very useful learning tool [5].

Scrape smear cytology is a modification of imprint cytology in which cells are harvested by scraping the cut surface of the specimen. It is an economical, simple and quick method of intra-operative diagnosis with acceptable sensitivity and high specificity, and does not alter the utility of the specimen for subsequent histopathology examination [6]. It can be used as an adjunct to frozen section and its routine utilization in ovarian lesions could aid in expanding the knowledge of cytology of ovarian neoplasms [7].

The aim of this work is to compare between intra-operative frozen section and scrape smear cytology in the diagnosis of ovarian neoplasm in Zagazig University Hospital.

\section{Patients and Methods}

This study was performed between March 2011 and March 2012, on 50 patients presented with ovarian mass recruited from the gynecology outpatient clinic. Patients with the following criteria were included: 1) clinically benign looking tumors (on preoperative radiology or intraoperative inspection) with raised CA 125; 2) adnexal mass in a patient with a past history of malignancy at another site; 3) young patients with ovarian neoplasms in whom fertility sparing surgery was planned; and 4) adnexal mass in any perimenopausal women.

The following was done for all selected patients: full history; full clinical examination and measurement of serum CA 125. The preoperative data studded were patient age, rapid weight loss, clinical examination, available imaging studies either ultrasonography, Computerized Tomography or both, level of the available tumor marker such as serum CA 125).

Patients with benign-appearing tumors at surgery underwent either cystectomy or salpingo-oophorectomy, 
depending on patient sand tumor characteristics. Patients with suspicious ovarian tumors underwent salpingooophorectomy. The intraoperative findings studded were presence of ascitis, adhesions to the adjacent structures, bilaterality, tumor implants on peritoneal surface, lymph node enlargement and gross picture of the tumor (e.g. tumor diameters, consistency, outer surface, cut surface, presence of necrotic areas and papillary structures within the cyst ).

The unfixed fresh specimen of the tumor was immediately delivered to Pathology Department-Faculty of Medicine-Zagazig University - with all the clinical details of the patient for evaluation. Gross examination of the tumor removed was done by inspection, palpation, The specimen was then cut with a sharp knife into two halves. The cut surface was wiped off the excess blood, if present, with the help of a filter paper. Again, reinspection and repalpation of the tumor was done. The most appropriate area thought to be representative of lesion was chosen.

The area was scraped with a sharp scalpel or the end of a glass slide, depending upon the type of tissue. A semifluid drop thus obtained was spread over a glass slide. One to four slides per case were taken from different representative areas. The slides were labelled and immediately put into 95\% ethyl alcohol and stained with hematoxylin-eosin, then frozen sections was obtained from the same areas of the scraping.

The specimens were then fixed in formalin. Sections were taken from the same area from where scrapings were taken. Paraffin blocks of the sections were processed in the routine way and sections were stained with hematoxylin and eosin ( $\mathrm{H}$ and $\mathrm{E})$.

All the sections studied microscopically under low and high power and categorized according to the status of malignancy into benign, borderline and malignant tumor.

The diagnosis obtained by intraoperative scrape and frozen section based on cellularity and cell morphology were compared with final histopathological diagnosis in terms of diagnostic sensitivity, to differentiate between benign and malignant lesions.

Assessment of the overall accuracy of the intraoperative diagnosis was classified as follows:

- Concordant. In these cases, the diagnosis was correct with regard to the major tumor category (benign, borderline, or malignant) and, in the case of malignant neoplasms, to tumor origin (primary vs metastatic).

- Discordant. These were cases in which incorrect or equivocal assessment could have adversely affected intraoperative management, including the failure to identify the principle diagnostic category of surface epithelial tumors (benign, borderline, or malignant) or inaccuracy regarding the origin of malignant neoplasms (primary vs metastatic). Cases in which the major histological category of primary ovarian malignancy (carcinoma, sex cord stromal tumor, and germ cell tumor) was not recognized were also considered discordant because the optimal operative management of these cases may differ, particularly in the younger age group.

Statistical analysis was performed using STATA 11.0 (College Station, Texas, USA). Data were presented as a number (\%) or mean \pm standard deviation (SD)/median (range). The differences in Mean/Median values were measured by using Student's t-test (normal data distribution)/Wilcoxon Ranksum Test (for categorical variables). $\mathrm{p}<0.05$ was considered to be statistically significant.

The study was approved by Zagazig University Medical Research Council Laboratories Joint Ethics Committee. All participants signed an informed consent. Rigorous confidentiality was maintained.

\section{Results}

Different types of ovarian masses (benign, bordreline and malignant) shows statistical significant correlation with the age of the patient (p value: 0.03) while there is no significant association between parity and different types of ovarian masses (p value: 0.25 ) (Table 1 \& Table 2). There was no statistically significant difference in the studied patients as regard the clinical data, macroscopic and intraoperative picture (p value: 0.8 ), while there was statistically significant association as regard the laterality of the ovarian masses (p value: 0.017 ). Table 3 shows the histological types of the tumors according to the results of paraffin sections, their number and percentage among patients under the study.

The sensitivity, specificity, positive predictive value, negative predictive value and accuracy of scraping technique in the diagnosis of benign ovarian masses were 100\%, 95.2\%, 96.7\%, 100\% and 98\% respectively. The sensitivity, specificity, positive predictive value, negative predictive value and accuracy of scraping technique in the diagnosis of border line ovarian masses were 100\%, 93.4\%, 25\%, 100\% and 94\% respectively. The sensitivity, specificity, positive predictive value, negative predictive value and accuracy of scraping technique in the diagnosis of malignant ovarian masses were $80 \%, 100 \%, 100 \%, 88.2 \%$ and $92 \%$ respectively. 
Table 1. Features of the studied patients (Age and parity) in relation to the type of ovarian masses ((benign, borderline and malignant).

\begin{tabular}{|c|c|c|c|c|c|}
\hline & $\begin{array}{c}\text { Benign } \\
(\mathrm{n}=29)\end{array}$ & $\begin{array}{l}\text { Borderline } \\
(\mathrm{n}=1)\end{array}$ & $\begin{array}{c}\text { Malignant } \\
(\mathrm{n}=20)\end{array}$ & $\mathbf{F}$ & $\mathbf{p}$ \\
\hline \multicolumn{6}{|l|}{ Age (years) } \\
\hline Mean \pm SD & $36.5 \pm 17.7$ & $43 \pm 0$ & $48.9 \pm 12.7$ & 3.57 & 0.03 \\
\hline Range & $11-70$ & & $19-77$ & & \\
\hline \multicolumn{6}{|l|}{ Parity } \\
\hline 0 & 13 (44.8\%) & $1(100 \%)$ & $6(30 \%)$ & & \\
\hline $1-3$ & 7 (24.1\%) & $0(0 \%)$ & $10(50 \%)$ & $X^{2}=5.06$ & 0.28 \\
\hline$>3$ & $9(31 \%)$ & $0(0 \%)$ & 4 (20\%) & & \\
\hline
\end{tabular}

Table 2. Association between different types of ovarian masses and clinical data, macroscopic \& intraoperative picture, and laterality of ovarian masses.

\begin{tabular}{|c|c|c|c|c|c|c|c|c|}
\hline & \multicolumn{2}{|c|}{ Benign } & \multicolumn{2}{|c|}{ Borderline } & \multicolumn{2}{|c|}{ Malignant } & \multirow{2}{*}{$\mathbf{X}^{2}$} & \multirow{2}{*}{$\mathbf{p}$} \\
\hline & No & $\%$ & No & $\%$ & No & $\%$ & & \\
\hline Clinical & 21 & 42.0 & 0 & 0.0 & 29 & 58.0 & \multirow{2}{*}{0.04} & \multirow{2}{*}{0.8} \\
\hline Macroscopic and intraoperative picture & 20 & 40.0 & 0 & 0.0 & 30 & 60.0 & & \\
\hline \multicolumn{9}{|l|}{ Laterality } \\
\hline Unilateral & 20 & 69.0 & 1 & 100.0 & 6 & 30.0 & \multirow{2}{*}{8.1} & \multirow{2}{*}{0.017} \\
\hline Bilateral & 9 & 31.0 & 0 & 0.0 & 14 & 70.0 & & \\
\hline
\end{tabular}

Table 3. The results of paraffin sections.

\begin{tabular}{|c|c|c|c|}
\hline Histologic type & Number of cases & $(\%)$ & N B: \\
\hline \multicolumn{4}{|l|}{ A. Epithelial tumors: } \\
\hline \multirow{3}{*}{$\begin{array}{c}\text { Benign: } \\
\text { Serous }\end{array}$} & & & \\
\hline & 8 cases & & 3/8 complicated \\
\hline & & $16 \%$ & *5/8 noncomplicated \\
\hline \multirow{2}{*}{ Mucinous } & 3 cases & & ${ }^{*} 1 / 8$ bilateral \\
\hline & & $6 \%$ & *1/3 complicated \\
\hline \multirow{3}{*}{ Brenner. } & & & ${ }^{*} 2 / 3$ non complicated \\
\hline & 1 case & & *1/3 bilateral \\
\hline & & $2 \%$ & Unilateral \\
\hline \multicolumn{4}{|l|}{ *Malignant. } \\
\hline Serouscyst adenoarcinoma & 12 case & $24 \%$ & *4/12 Serouscyst adenoarcinoma, 3/4 bilateral. \\
\hline & & & "8/12 papillary Serouscyst adenoarcinoma,6/8 bilat \\
\hline Endometrioid adenocarinoma & 6 cases & $12 \%$ & *3/6 bilateral \\
\hline \multicolumn{4}{|l|}{ B. Sex-cord stromal neoplasm: } \\
\hline Fibrothecoma & 4 cases & $8 \%$ & Unilat \\
\hline Fibroma & 1 case & $2 \%$ & Unilat \\
\hline \multicolumn{4}{|l|}{ C. Germcell neoplasm: } \\
\hline Teratoma & 5 cases & $10 \%$ & 2/5 bilateral \\
\hline Dysgerminoma. & 2 cases & $4 \%$ & 1/2 bilateral \\
\hline D. Metastatic (Krukenberg): & 1 case & $2 \%$ & Bilateral \\
\hline E. Others: & & & $4 / 5$ bilateral \\
\hline Endometrioti cyst & 5 cases & $10 \%$ & 1 non specific. \\
\hline Inflammatory cyst & 2 cases & $4 \%$ & 1 TB granuloma (bilateral) \\
\hline
\end{tabular}




\section{A. M. Abdelghany et al.}

The sensitivity, specificity, positive predictive value, negative predictive value and accuracy of frozen section in the diagnosis of benign ovarian masses were $100 \%, 100 \%, 100 \%, 100 \%$ and $100 \%$ respectively. The sensitivity, specificity, positive predictive value, negative predictive value and accuracy of frozen section in the diagnosis of borderline ovarian masses were 100\%, 95.9\%, 33.3\%, 100\% and 96\% respectively. The sensitivity, specificity, positive predictive value, negative predictive value and accuracy of frozen section in the diagnosis of malignant ovarian masses were $90 \%, 100 \%, 100 \%, 93.8 \%$ and 96\% respectively (Tables 4-7).

Table 4. Comparison between scraping and paraffin section according to the status of malignancy.

\begin{tabular}{|c|c|c|c|c|}
\hline & \multicolumn{3}{|c|}{ Paraffin section } & \multirow{2}{*}{ Total } \\
\hline & Benign & Borderline & Malignant & \\
\hline \multicolumn{5}{|l|}{ Scraping } \\
\hline Benign & 29 & 0 & 1 & 30 \\
\hline Borderline & 0 & 1 & 3 & 4 \\
\hline Malignant & 0 & 0 & 16 & 16 \\
\hline Total & 29 & 1 & 20 & 50 \\
\hline
\end{tabular}

Table 5. Validity of scraping in the diagnosis of ovarian masses in relation to parafin section.

\begin{tabular}{cccc}
\hline & Benign & Borderline & Malignant \\
\hline Sensitivity & 100.0 & 100.0 & 80.0 \\
Specificity & 95.2 & 93.9 & 100.0 \\
Positive predictive value & 96.7 & 25.0 & 100.0 \\
Negative predictive value & 100.0 & 100.0 & 88.2 \\
Accuracy & 98.0 & 94.0 & 92.0 \\
\hline
\end{tabular}

Table 6. Comparison between frozen section and paraffin section according to the status of malignancy.

\begin{tabular}{ccccc} 
& & Paraffin section & Total \\
\cline { 2 - 3 } & Benign & Borderline & Malignant & \\
Frozen section & & & 0 & 3 \\
Benign & 29 & 0 & 2 & 18 \\
Borderline & 0 & 1 & 18 & 50 \\
Malignant & 0 & 0 & 20 & 30 \\
Total & 29 & 1 & & \\
\hline
\end{tabular}

Table 7. Validity of frozen section in the diagnosis of ovarian masses in relation to parafin section.

\begin{tabular}{cccc}
\hline & Benign & Borderline & Malignant \\
\hline Sensitivity & 100.0 & 100.0 & 90.0 \\
Specificity & 100.0 & 95.9 & 100.0 \\
Positive predictive value & 100.0 & 33.3 & 100.0 \\
Negative predictive value & 100.0 & 100.0 & 93.8 \\
Accuracy & 100.0 & 96.0 & 96.0 \\
\hline
\end{tabular}




\section{Discussion}

Rapid intra-operative diagnosis of the nature of ovarian tumors is both interesting and challenging for effective planning of the surgical management of these tumors, particularly in a young woman as it can avoid unnecessary removal of contralateral ovary and helps preserve fertility [8].

Intraoperative frozen section for use in diagnosis of ovarian tumors is of great value. In some cases, it can help surgeons avoid under-treatment or overtreatment of patients. Frozen section is indicated to ensure that the tissue sampled adequate for diagnosis, to determine the nature of the disease process, to plan ancillary studies, to determine tumor spread, and to assess margins [9]. Most studies have shown that if ovarian tumors are categorized according to their malignant potential, the accuracy of frozen section diagnosis would be in the range of $89.8 \%$ - 97\% [10].

In this study, the sensitivity, specificity, positive predictive value, negative predictive value and accuracy of frozen section in the diagnosis of benign ovarian masses were $100 \%, 100 \%, 100 \%, 100 \%$ and $100 \%$ respectively. The sensitivity, specificity, positive predictive value, negative predictive value and accuracy of frozen section in the diagnosis of borderline ovarian masses were $100 \%, 95.9 \%, 33.3 \%, 100 \%$ and $96 \%$ respectively. The sensitivity, specificity, positive predictive value, negative predictive value and accuracy of frozen section in the diagnosis of malignant ovarian masses were $90 \%, 100 \%, 100 \%, 93.8 \%$ and $96 \%$ respectively.

In a systematic review of papers examining the use of intraoperative frozen section in predicting the final diagnosis of ovarian lesions, the sensitivity of frozen section was greater than $95 \%$ when benign tumors were compared with borderline and invasive carcinomas but just less than this when borderline and invasive carcinomas were compared. Specificity also exceeded $95 \%$ when benign and invasive carcinoma were compared but was less than this when borderline lesions were compared firstly with benign and secondly with invasive lesions. The positive and negative predictive values were also optimal when benign and invasive carcinomas were compared but reduced when either benign or invasive carcinomas were compared with borderline lesions [11].

Ilker et al., analyzed data of 266 patients with ovarian masses. The results of frozen sections were 235 (88.3\%) benign, 11 (4.2\%) borderline and 20 (7.5\%) malignant. The sensitivity was $100 \%$ for the benign tumors, $77.8 \%$ for the borderline tumors and $71.4 \%$ for the malignant tumors. The specificity of frozen section was $83.8 \%, 98.4 \%$ and $100 \%$ for the benign, borderline and malignant tumors, respectively [12].

Wootipoom et al. concluded that intraoperative frozen section diagnosis is generally accurate and can be used as one piece of evidence for the surgeon to use in determining the type and extent of initial surgery to be performed. However, frozen section has limitations such as sampling error, deferred diagnosis and interpretation error. Good intraoperative communication between surgeons and pathologists and regular clinico-pathologic conferences, especially in cases with discordant diagnosis, can maximize accuracy and minimize limitations such as interpretation error and deferred diagnosis [13]. According to Stewart et al., several factors can affect the accuracy of frozen section diagnosis, including sampling error, quality of frozen section (technical factor), and experience of pathologists [14]. Sampling error was considered to be the main reason for the diagnostic discrepancy in most studies, predominantly in some groups of tumors, in which there was remarkable heterogeneity of tumor from areas to areas within the same ovarian mass, such as mucinous tumors and teratomas. Frozen section may fail to sample the most severe lesion or frankly malignant area in a limited number of sections intraoperatively [15]. Another factor that causes difficulty in frozen section diagnosis is the quality of sections prepared by this technique that limits the evaluation of cellular detail. This technical factor is rather underemphasized in most clinical studies on frozen section diagnosis [16]. The section quality was a reason of deferred or discordant diagnoses in this study; also it caused difficulty in some cases of primary epithelial carcinoma in the agreement group with paraffin diagnosis. However, such limitation did not result in the alteration of the intra-operative surgical management in our cases.

Commonly used methods for obtaining and preparing cells for cytological evaluation are touch preparation, fine needle aspiration cytology (FNAC) and scrape smear preparation [17].

Cytological evaluation provides a better morphological detail, and in experienced setup compares well with frozen section and subsequent formalin-fixed paraffin-embedded sections. In addition, cytological examination of surgical specimens has proved to be a valuable learning tool and has educational value. This significant educational value coupled with its intrinsic simplicity and rapidity and cost effectiveness will likely necessitate the widespread implementation of this diagnostic technique in the near future [18]. The cytological preparations were also advantageous when sampling of multiple sites was required because this helped to confirm the presence of tumor dissemination without the requirement for additional frozen sections. Finally, and despite excellent 
technical support, there were occasions in which the frozen section slides were suboptimal because of necrosis, hemorrhage, calcification, non-representative sampling, or other technical factors, and in these cases, the cytology preparations frequently provided better cellular detail for diagnosis [17]. Material obtained by this technique can be utilized for flow cytometry and cytogenetic studies. In spite of the various applications, its use has not been widely recognized in diagnosis of ovarian tumors [5].

Scrape cytology could be preferred over touch preparation/ imprint cytology, as in most cases, the former technique would yield much more material than the latter. Further, its role as a potential tool in intra-operative consultation is more pertinent in institutions unequipped with frozen section facility. In these scenarios, in experienced hands, it not only offers a viable alternative, but also helps to reduce the overall cost and processing time, without compromising quality [3].

History of scrape cytology can be traced back to 1927 when Leonard S. Dudgeon and Vincent Patrick at the University of London raised the horizons of the rapid cytological diagnosis of freshly cut specimens with reliable accuracy rates. Following this, several studies done in the past have discussed the use of imprint and touch preparation, especially as a tool for intraoperative diagnosis [19]. After these initial trials, the use of cytology smears during intraoperative consultation has often been neglected in favor of traditional examination of frozen sections. This appears to be due to the surgical pathologist's relatively higher level of confidence in frozen sections, though many studies have demonstrated that the diagnostic efficacy of intraoperative cytology is comparable to that of frozen section [20]. So, this study was undertaken to know the utility of scrape cytology in the intraoperative diagnosis of tumor.

We obtained very good results while using scrape cytology. In this study, the sensitivity, specificity, positive predictive value, negative predictive value and accuracy of scraping technique in the diagnosis of benign ovarian masses were $100 \%, 95.2 \%, 96.7 \%, 100 \%$ and $98 \%$ respectively. The sensitivity, specificity, positive predictive value, negative predictive value and accuracy of scraping technique in the diagnosis of border line ovarian masses were $100 \%, 93.4 \%, 25 \%, 100 \%$ and $94 \%$ respectively. The sensitivity, specificity, positive predictive value, negative predictive value and accuracy of scraping technique in the diagnosis of malignant ovarian masses were $80 \%, 100 \%, 100 \%, 88.2 \%$ and $92 \%$ respectively.

Shidham et al. [21] and Khunamornpong et al. [3] observed that scraping of tumor is the method preferred because large number of cells can be obtained and cells can be spread well on the slides. According to Esteban et al. [22] touch preparation yields less cellular smears than scrape smears. Although the cytology smears proved useful in many cases, Stewart et al. [14] found that frozen section had a greater sensitivity than intraoperative cytology in the diagnosis of malignant ovarian lesions ( $97 \%$ vs $93 \%$, respectively), although the specificity of both techniques for a malignant diagnosis was $100 \%$. These findings are similar to this study.

\section{Conclusion}

Finally, we can conclude that frozen section proved to be more accurate than smear preparations in the intraoperative assessment of ovarian tumors in this study. However, the cytology preparations were helpful in supporting the histological diagnoses, and in some cases, provided additional useful information. Thus, cytology has a complementary role to frozen section in the intraoperative assessment of ovarian lesions. At the centers where the facilities of frozen section are not available, intraoperative scrape cytology is a useful tool for intraoperative diagnosis of tumor. It can be used to diagnose small tissue that can be preserved for permanent paraffin block method.

\section{References}

[1] DiSaia, P.J. and Creasman, W.T. (2008) The Adnexal Mass and Early Ovarian Cancer. 7th Edition, Clinical Gynecologic Oncology, Mosby, Chapter 10, 283-312.

[2] Stewart, C.J., Brennan, B.A., Hammond, I.G., Leung, Y.C. and McCartney, A.J. (2006) Intraoperative Assessment of Ovarian Tumors: A 5-Year Review with Assessment of Discrepant Diagnostic Cases. International Journal of Gynecological Pathology, 25, 216-222.

[3] Khunamornpong, S. and Siriaunkgul, S. (2003) Scrape Cytology of the Ovaries: Potential Role in Intraoperative Consultation of Ovarian Lesions. Diagnostic Cytopathology, 28, 250-257. http://dx.doi.org/10.1002/dc.10273

[4] Alvarez Santín, C., Sica, A., Melesi, S., Feijó, A., Garrido, G. and Rodríguez Alvarez, C. (2011) Contribution of Intraoperative Cytology to the Diagnosis of Ovarian Lesions. Acta Cytologica, 55, 85-91.

http://dx.doi.org/10.1159/000320859 
[5] Stewart, C.J., Brennan, B.A., Koay, E., Naran, A. and Ruba, S. (2010) Value of Cytology in the Intraoperative Assessment of Ovarian Tumors: A Review of 402 Cases and Comparison with Frozen Section Diagnosis. Cancer Cytopathology, 118, 127-136. http://dx.doi.org/10.1002/cncy.20073

[6] Kolte, S.S. and Satarkar, R.N. (2010) Role of Scrape Cytology in the Intraoperative Diagnosis of Tumor. Journal of Cytology, 27, 86-90. http://dx.doi.org/10.4103/0970-9371.71871

[7] Deb, P., Malik, A. and Sinha, K.K. (2011) Intraoperative Scrape Cytology: Adult Granulosa Cell Tumor of Ovary. Journal of Cytology, 28, 207-209. http://dx.doi.org/10.4103/0970-9371.86350

[8] Kar, T., Kar, A. and Mohapatra, P.C. (2005) Intra-Operative Cytology of Ovarian Tumors. The Journal of Obstetrics and Gynecology of India, 55, 345-349.

[9] Ilvan, S., Ramazanoglu, R., Ulker Akyildiz, E., et al. (2005) The Accuracy of Frozen Section (Intraoperative Consultation) in the Diagnosis of Ovarian Masses. Gynecologic Oncology, 97, 395-399. http://dx.doi.org/10.1016/j.ygyno.2005.01.037

[10] Geomini, P., Bremer, G., Kruitwagen, R., et al. (2005) Diagnostic Accuracy of Frozen Section Diagnosis of the Adnexal Mass: A Meta-Analysis. Gynecologic Oncology, 96, 1-9. http://dx.doi.org/10.1016/j.ygyno.2004.09.042

[11] Heatley, M.K. (2012) A Systematic Review of Papers Examining the Use of Intraoperative Frozen Section in Predicting the Final Diagnosis of Ovarian Lesions. International Journal of Gynecological Pathology, 31, 111-115. http://dx.doi.org/10.1097/PGP.0b013e318226043b

[12] Ilker, A., Aykut, B., Muge, H., Ibrahim, H.M., Ulku, O.B., Sener, G., et al. (2011) Accuracy of Intra-Operative Frozen Section in the Diagnosis of Ovarian Tumors. Journal Pakistan Medical Association, 61, 856-858.

[13] Wootipoom, V., Dechsukhum, C., Hanprasertpong, J., et al. (2006) Accuracy of Intra-Operative Frozen Section in Diagnosis of Ovarian Tumors. Journal of the Medical Association of Thailand, 89, 577-582.

[14] Stewart, C.J., Brennan, B.A., Koay, E., Naran, A. and Ruba, S. (2010) Value of Cytology in the Intraoperative Assessment of Ovarian Tumors: A Review of 402 Cases and Comparison with Frozen Section Diagnosis. Cancer Cytopathology, 118, 127-136. http://dx.doi.org/10.1002/cncy.20073

[15] Tempfer, C.B., Polterauer, S., Bentz, E.K., Reinthaller, A. and Hefler, L.A. (2007) Accuracy of Intra-Operative Frozen Section Analysis in Borderline Tumors of the Ovary: A Retrospective Analysis of 96 Cases and Review of the Literature. Gynecologic Oncology, 107, 248-252. http://dx.doi.org/10.1016/j.ygyno.2007.06.008

[16] Brun, J.L., Cortez, A., Rouzier, R., et al. (2008) Factors Influencing the Use and Accuracy of Frozen Section Diagnosis of Epithelial Ovarian Tumors. American Journal of Obstetrics and Gynecology, 199, 244. http://dx.doi.org/10.1016/j.ajog.2008.04.002

[17] Coffey, D., Kaplan, A.L. and Ramzy, I. (2005) Intraoperative Consultation in Gynecologic Pathology. Archives of Pathology and Laboratory Medicine, 129, 1544-1557.

[18] Rao, S., Sadiya, N., Joseph, L.D. and Rajendiran, S. (2009) Role of Scrape Cytology in Ovarian Neoplasms. Journal of Cytology, 26, 26-29. http://dx.doi.org/10.4103/0970-9371.54864

[19] Gal, R. (1988) Scrape Cytology Assessment of Margins of Lumpectomy Specimens in Breast Cancer. Acta Cytologica, 32, 838-839.

[20] Mair, S., Lash, R.H., Suskin, D. and Mendelsohn, G. (1991) Intraoperative Surgical Specimen Evaluation: Frozen Section Analysis, Cytologic Examination, or Both? American Journal of Clinical Pathology, 96, 8-14.

[21] Shidham, V.B., Dravid, N.V., Grover, S. and Kher, A.V. (1984) Role of Scrape Cytology in Rapid Intraoperative Diagnosis: Value and Limitations. Acta Cytologica, 28, 477-482.

[22] Esteban, J.M., Zaloudek, C. and Silverberg, S.G. (1987) Intraoperative Diagnosis of Breast Lesion: Comparison of Cytologic with Frozen Section Technics. American Journal of Clinical Pathology, 88, 681-688. 
Scientific Research Publishing (SCIRP) is one of the largest Open Access journal publishers. It is currently publishing more than 200 open access, online, peer-reviewed journals covering a wide range of academic disciplines. SCIRP serves the worldwide academic communities and contributes to the progress and application of science with its publication.

Other selected journals from SCIRP are listed as below. Submit your manuscript to us via either submit@scirp.org or Online Submission Portal.
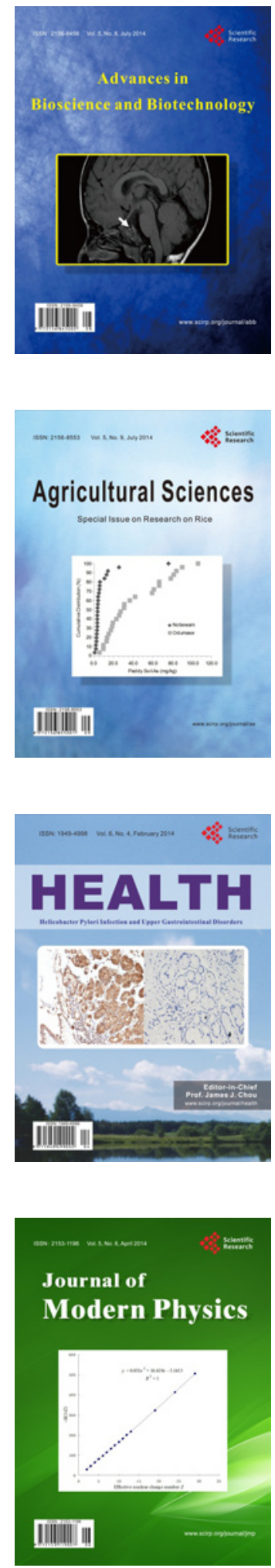
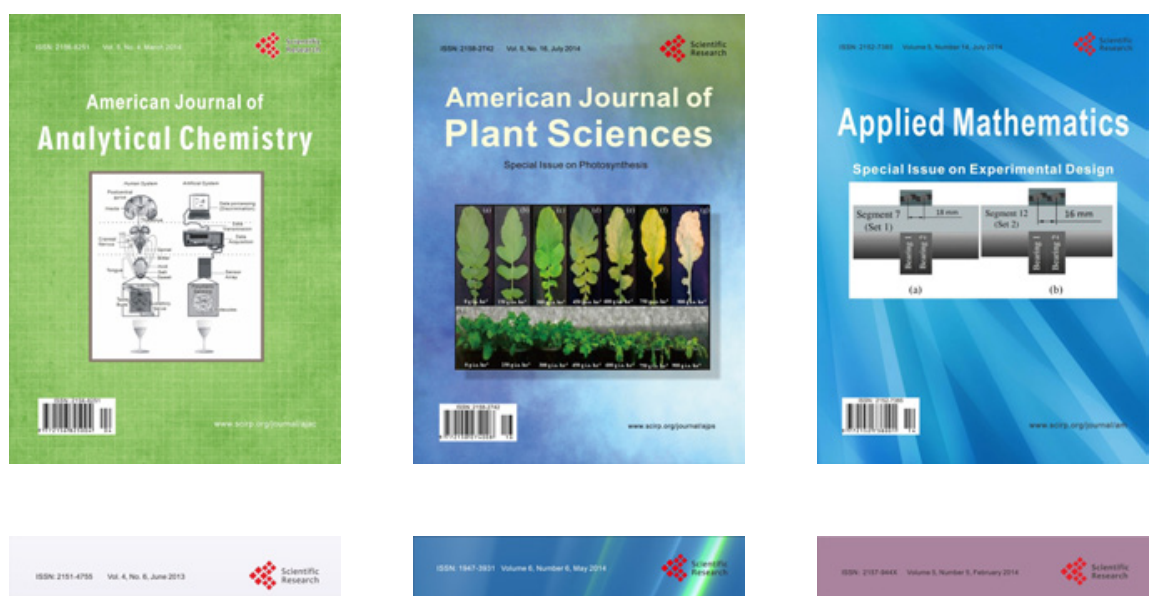

Creative Education
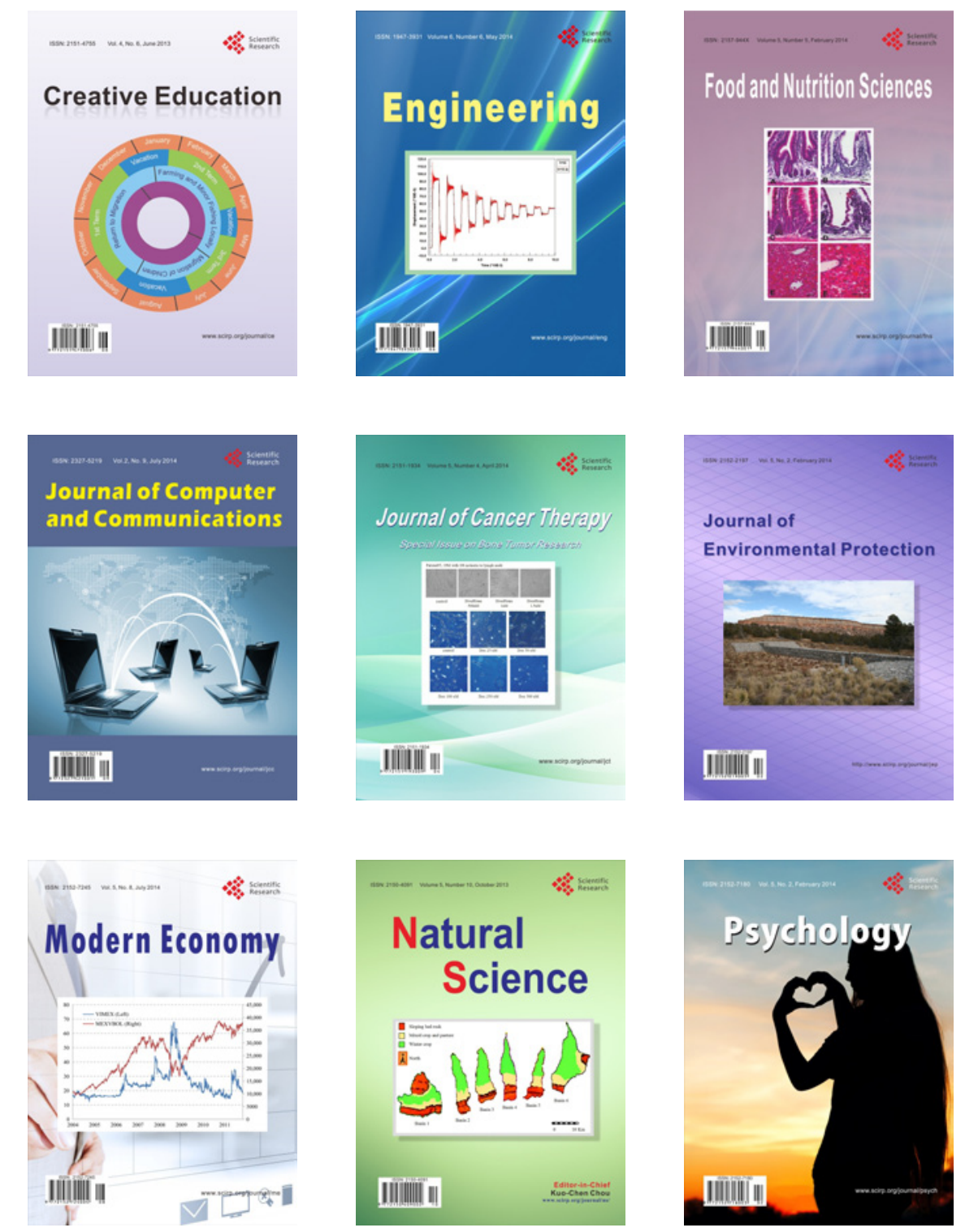